\author{
Marquette University \\ e-Publications@Marquette
}

College of Nursing Faculty Research and

Publications

Nursing, College of

\title{
8-2011
}

\section{Comparison of the Effects of Ice and 3.5\% Menthol Gel on Blood Flow and Muscle Strength of the Lower Arm}

\author{
Robert V. Topp \\ Marquette University, robert.topp@marquette.edu \\ Lee J. Winchester \\ University of Louisville \\ Amber M. Mink \\ University of Louisville \\ Jeremiah S. Kaufmann \\ University of Louisville \\ Dean E. Jacks \\ University of Louisville
}

Follow this and additional works at: https://epublications.marquette.edu/nursing_fac

Part of the Nursing Commons

\section{Recommended Citation}

Topp, Robert V.; Winchester, Lee J.; Mink, Amber M.; Kaufmann, Jeremiah S.; and Jacks, Dean E., "Comparison of the Effects of Ice and 3.5\% Menthol Gel on Blood Flow and Muscle Strength of the Lower Arm" (2011). College of Nursing Faculty Research and Publications. 278.

https://epublications.marquette.edu/nursing_fac/278 


\title{
Comparison of the Effects of Ice and 3.5\% Menthol Gel on Blood Flow and Muscle Strength of the Lower Arm
}

\author{
Robert Topp, Lee Winchester, Amber M. Mink, \\ Jeremiah S. Kaufman, and Dean E. Jacks
}

\begin{abstract}
Context: Soft-tissue injuries are commonly treated with ice or menthol gels. Few studies have compared the effects of these treatments on blood flow and muscle strength. Objective: To compare blood flow and muscle strength in the forearm after an application of ice or menthol gel or no treatment. Design: Repeatedmeasures design in which blood-flow and muscle-strength data were collected from subjects under 3 treatment conditions. Setting: Exercise physiology laboratory. Participants: 17 healthy adults with no impediment to the blood flow or strength in their right arm, recruited through word of mouth. Intervention: Three separate treatment conditions were randomly applied topically to the right forearm: no treatment, $0.5 \mathrm{~kg}$ of ice, or $3.5 \mathrm{~mL}$ of $3.5 \%$ menthol gel. To avoid injury ice was only applied for $20 \mathrm{~min}$. Main Outcome Measures: At each data-collection session blood flow $(\mathrm{mL} / \mathrm{min})$ of the right radial artery was determined at baseline before any treatment and then at 5,10, 15, and 20 min after treatment using Doppler ultrasound. Muscle strength was assessed as maximum isokinetic flexion and extension of the wrist at $30 \% \mathrm{~s} 20,25$, and $30 \mathrm{~min}$ after treatment. Results: The menthol gel reduced $(-42 \%, P<.05)$ blood flow in the radial artery $5 \mathrm{~min}$ after application but not at 10,15 , or $20 \mathrm{~min}$ after application. Ice reduced $(-48 \%, P$ $<.05$ ) blood flow in the radial artery only after $20 \mathrm{~min}$ of application. After 15 min of the control condition blood flow increased $(83 \%, P<.05)$ from baseline measures. After the removal of ice, wrist-extension strength did not increase per repeated strength assessment as it did during the control condition $(9-11 \%, P<$ $.05)$ and menthol-gel intervention $(8 \%, P<.05)$. Conclusions: Menthol has a fast-acting, short-lived effect of reducing blood flow. Ice reduces blood flow after a prolonged duration. Muscle strength appears to be inhibited after ice application.
\end{abstract}

Keywords: cryotherapy, vascular response, strength

Athletic soft-tissue injuries that occur during practice or competition are commonly accompanied by inflammation and pain and in combination contribute to

The authors are with the Dept of Exercise Physiology, University of Louisville, Louisville, KY. 
declines in muscle strength. Various topical therapies have been employed in an attempt minimize pain and enhance muscle strength after soft-tissue injury. ${ }^{1-3}$ These therapies including the application of ice and menthol gels have been employed by athletic trainers, physical therapists, and other health care providers in an attempt to minimize the injury and return the individual to training or competition as soon as possible. ${ }^{4}$

The cooling effect of menthol has also been postulated to affect blood flow, although there are limited data to support this claim. In a study of decerebrate cats the application of topical menthol gel reduced the exercise-pressor effect (blood flow) within an activated muscle without a systemic effect on heart rate. ${ }^{5}$ Those authors and others examining topical analgesic gels have reported that increases in blood pressure are attenuated during exercise, probably because of an increase in total peripheral resistance at the muscle area, ${ }^{5,6}$ thus leading to a reduction in overall blood flow to the area. It is thought that the reduction in the pressor response is caused by the inhibition of the small-diameter sensory nerve fibers that are known as group III and IV afferents that synapse with the central nervous system. ${ }^{5}$ Those authors concluded that topical menthol may reduce blood flow to the underlying tissues through stimulation of cold receptors of the skin.

Topical gels that contain menthol also result in a cooling sensation to the area where the gel is applied and are commonly used by health care providers including athletic trainers and physical therapists to treat soft-tissue injuries. Topical menthol gels are classified as topical analgesics with a mechanism of action defined as counterirritant, based on the gate control theory. ${ }^{7}$ Menthol gels are thought to produce a cooling sensation by inhibiting calcium currents of neuronal membrane. ${ }^{8}$ Menthol is noted for eliciting perceptions of cold through its effects on the transient-receptor-potential (TRP) family of ion channels. Haeseler et $\mathrm{al}^{9}$ postulated that the stimulating effect of menthol on cold receptors may have an antinociceptive effect causing an inactivation of sodium channels of the alpha subunit, resulting in hyperpolarization or blocking the signal of pain transduction or impulses from pain receptors. Another study demonstrating the antinociceptive effects of menthol in mice on a hotplate suggested that the temporary pain relief from menthol was as effective as morphine for the hotplate test. ${ }^{8}$

Despite the fact that menthol gels and ice are commonly used to treat soft-tissue injuries, little empirical study has been conducted to compare menthol gels with the application of ice. Thus, the purpose of this study was to compare blood flow and muscle strength in the forearm after an application of ice or $3.5 \%$ menthol gel (Biofreeze) or a no-treatment control condition. This purpose was addressed by evaluating the tenability of the following hypotheses:

H1: The application of ice or $3.5 \%$ menthol gel to the forearm would result in a decrease in blood flow to the forearm compared with a no-treatment control condition.

$\mathrm{H} 2$ : The application of ice or $3.5 \%$ menthol gel to the forearm would result in different forearm muscle strength compared with a no-treatment control condition. 


\section{Methods}

\section{Design}

The design of the study to address the hypotheses used repeated measures in which blood-flow and muscle-strength data were collected from subjects on 3 separate days. The data-collection protocol on each of these days was the same except for the treatment intervention that was applied. Before any data collection, subjects read and signed an informed consent that was approved by the University of Louisville's institutional review board. After we obtained consent all subjects completed a brief demographic questionnaire, and height, weight, and body composition were measured. Immediately after providing consent all subjects completed a familiarization trial with the data-collection protocol to minimize any novelty effect when study data were collected. The order of the treatments (menthol, ice, and control) was counterbalanced to be administered to subjects during each of the 3 subsequent data-collection sessions. These 3 data-collection sessions were separated by at least 7 days to provide a washout period between treatments. Each data-collection session included blood-flow measurements at 5 different times followed by assessments of strength at 3 different times.

\section{Participants}

Seventeen healthy adults ( 9 men and 8 women, mean age 24.4 y) were recruited to participate in the study through word of mouth. Subjects reported no health conditions that may have altered the blood flow or strength in their right arm (diabetes, Reynaud's disease, peripheral vascular disease, etc). None of the subjects smoked and all refrained from caffeine consumption for 8 hours before data collection. The sample averaged 136 minutes of aerobic exercise and 149 minutes of strength training per week and exhibited normal ranges of resting heart rate, blood pressure, and body composition (see Table 1).

Table 1 Demographic Characteristics of the Sample $(\mathrm{N}=17)$

\begin{tabular}{lcccc}
\hline Variable & Minimum & Maximum & Mean & SD \\
\hline Age (y) & 21 & 29 & 24.41 & 2.72 \\
Height (in) & 62 & 73 & 67.43 & 3.36 \\
Weight (lb) & 117 & 233 & 157.63 & 29.63 \\
Resting heart rate (beats/min) & 52 & 93 & 69.24 & 11.73 \\
Resting systolic blood pressure (mmHg) & 110 & 145 & 124.41 & 9.61 \\
Resting diastolic blood pressure (mmHg) & 55 & 91 & 78.18 & 8.52 \\
Percent body fat & 3.85 & 32.91 & 18.92 & 7.84 \\
Min/wk spent in aerobic exercise & 0 & 500 & 135.59 & 122.37 \\
Min/wk spent in strength-training exercise & 0 & 280 & 145.88 & 86.25 \\
\hline
\end{tabular}




\section{Procedures}

The data-collection procedure was developed to mimic clinical management of a soft-tissue injury. One of 3 randomly selected treatments (menthol, ice, or control) was applied to each subject's right forearm during each data-collection session. A common practice is to reassess an athlete for competition 20 minutes after immediate therapy for a soft-tissue injury. The intervention protocol involved application of 1 of the 3 treatments (menthol, ice, or control) for 20 minutes; blood flow was assessed before treatment application and 5, 10, 15, and 20 minutes afterward. After the blood-flow measurement collected 20 minutes after treatment application, muscle-strength tests were performed. If ice was the treatment applied it was removed after the 20-minute blood-flow assessment but before muscle strength was measured. Subjects performed an assessment of their muscle strength immediately after the final blood-flow assessment approximately 20, 25, and 30 minutes after application of the treatment after assessments of blood flow.

The duration of the treatment was 20 minutes to be consistent with the recommended duration of ice application for soft-tissue injuries. ${ }^{10}$ The application of the ice treatment involved $0.5 \mathrm{~kg}$ of $0^{\circ} \mathrm{C}$ ice obtained from a commercial ice machine. This ice was captured in a $2-\mathrm{L}$ plastic bag, applied without compression to the anterior surface of the forearm, and secured in place with Velcro straps. The dose of $3.5 \mathrm{~mL}$ of menthol gel was based on previous pilot testing and what is typically applied in athletic training facilities $\left(1 \mathrm{~mL}\right.$ of gel for every $200 \mathrm{~cm}^{2}$ of surface area). The dose of menthol gel was standardized from the average adult forearm presenting with approximately $700 \mathrm{~cm}^{2}$ of surface area. This standard amount $(3.5$ $\mathrm{mL}$ ) of menthol gel was applied by a gloved technician from the subject's wrist to his or her elbow on all surfaces of the right forearm. Menthol gel is absorbed or evaporates after 20 minutes and is not commonly removed after application. During the control condition no treatment was applied to the forearm during any of the data collection.

On each day of data collection subjects reported to the laboratory and were placed in a seated position on the device where all the data collection took place (Biodex 1000 isokinetic dynamometer) and told to rest quietly for 10 minutes before any data collection. Heart rate (beats/min) was palpated on the left side in the radial artery, and blood pressure ( $\mathrm{mmHg}$ ) measured using a manual mercury sphygmomanometer on the left arm immediately before all blood-flow measures. Blood flow was measured noninvasively in the right radial artery, proximal to the carpus between the radial collateral ligament of the wrist and the tendons of the abductor pollicis longus and extensor pollicis brevis, using high-resolution ultrasound (Philips HDI 5000, Seattle, WA). The radial artery was imaged longitudinally, by B-mode ultrasound, using a 12- to $5-\mathrm{mHz}$ linear-array transducer. This method of estimating blood flow is reported to be highly valid $(r=.96-.98)$ when calculating blood flow in different vessel compartments. ${ }^{11} \mathrm{~A}$ video file of the ultrasound was collected over 5 pulsations (or heartbeats) at the specified data-collection intervals in the vessel to allow data analysis after the test. A custom-made software program using LabView version 7.1 (National Instruments, Austin, TX) measured the changes in brachial diameter beat by beat and calculated a volume per beat passing through the vessel. These volumes were averaged over 5 consecutive beats and then multiplied by the heart rate at the time the volumes were collected to arrive 
at a volume per minute $(\mathrm{mL} / \mathrm{min})$ coursing through the radial artery at the specific data-collection time point.

With subjects in the initial seated position from which blood flow was measured and immediately after the final blood-flow measurement, muscle strength of the right forearm was assessed using a Biodex 1000 isokinetic dynamometer. Subjects were seated upright with restraints applied to their waist, torso, and upper and lower arm. The axis of rotation of the dynamometer was placed at the wrist, and subjects grasped a handle by which to extend and flex their wrist. Each subject's passive wrist-extension and -flexion range of motion was determined. Subjects were then allowed to familiarize themselves with the wrist-extension/flexion motion at a rotation speed of $30 \%$ s by performing 5 to 10 submaximal wrist extensions and flexions using the isokinetic dynamometer. They were then asked to complete 30 repeated maximum flexions and extensions of the wrist at a rotation speed of $30 \%$. The 5 highest torque recordings were averaged to arrive at a maximum wrist-extension and -flexion strength measure. This protocol was developed to assess the possible mitigating effects of ice and menthol on pain and fatigue resulting from the highintensity, short-duration exercise.

\section{Statistical Analysis}

A repeated-measures analysis of variance (R-ANOVA) using SPSS 16.0 was used to test for effects of time, treatment, and interaction (time by treatment) to address the 2 research hypotheses. A $3 \times 5$ (treatment $\times$ time) design was used to assess changes in blood flow, and a $3 \times 3$ (treatment $\times$ time) design was used to assess changes in muscle strength. Significant main effects were further evaluated by calculating Tukey least-significant post hoc differences. All analyses were considered statistically significant at $P<.05$.

\section{Results}

An interaction of time by group on blood flow $(F=8.15, P<.00)$ was indicated by the R-ANOVA. Post hoc analysis indicated that blood flow changed in different directions in each of the study groups at different time points after the application of the treatment. As Table 2 indicates, at 5 minutes after application of the menthol gel blood flow significantly declined in the radial artery by $42 \%$. This decline in blood flow was not sustained at 10, 15, or 20 minutes after the menthol-gel treatment. Under the control condition blood flow significantly increased by $83 \%$ at 15 minutes after baseline measures and then returned to baseline levels at 20 minutes after baseline measures. Finally, the ice treatment resulted in a significant decline $(48 \%)$ in blood flow in the radial artery only at 20 minutes after application of the therapy. All other blood-flow measures under the ice condition, at 5, 10, and 15 minutes, were not different than the baseline measure.

R-ANOVAs were also employed to evaluate the second study hypothesis. This statistic indicated no significant time $(F=0.45, P=.51)$, treatment $(F=0.81, P=$ $.83)$, or time-by-treatment $(F=0.21, P=.83)$ effect on wrist-flexion strength (see Table 3). The same statistic was applied to wrist-extension strength measures and indicated a significant effect of time $(F=11.07, P<.00)$ but no effect of treatment $(F=0.92, P<.41)$ or the interaction of treatment by time $(F=0.24, P<.79$; see 
Table 4). Post hoc analysis indicated that although no interaction of treatment and time emerged, only the menthol and the control conditions exhibited significant increases in wrist-extension strength at the 25- and 30-minute data-collection points compared with the strength measures obtained in the respective treatment condition at 20 minutes after treatment application. These increases ranged from

Table 2 Changes in Blood Flow Over 20 min by Treatment Condition

\begin{tabular}{llccc}
\hline \multirow{2}{*}{ Treatment } & \multicolumn{2}{c}{ Blood Flow $(\mathrm{mL} / \mathrm{min})$} & \\
\cline { 3 - 4 } Menthol & Time from baseline & Mean & SE & Change from baseline \\
\hline & baseline & 6.86 & 1.42 & - \\
& $5 \mathrm{~min}$ & $4.06^{*}$ & 1.40 & $-42 \%$ \\
& $10 \mathrm{~min}$ & 5.59 & 1.21 & $-19 \%$ \\
& $15 \mathrm{~min}$ & 7.18 & 1.54 & $5 \%$ \\
Control & $20 \mathrm{~min}$ & 6.41 & 1.21 & $-7 \%$ \\
& baseline & 5.01 & 1.42 & - \\
& $5 \mathrm{~min}$ & 6.78 & 1.40 & $35 \%$ \\
& $10 \mathrm{~min}$ & 7.17 & 1.21 & $43 \%$ \\
Ice & $15 \mathrm{~min}$ & $9.18 *$ & 1.54 & $83 \%$ \\
& $20 \mathrm{~min}$ & 5.93 & 1.21 & $18 \%$ \\
& baseline & 7.34 & 1.42 & - \\
& $5 \mathrm{~min}$ & 7.57 & 1.40 & $2 \%$ \\
& $10 \mathrm{~min}$ & 5.77 & 1.21 & $-22 \%$ \\
& $15 \mathrm{~min}$ & 5.91 & 1.54 & $-20 \%$ \\
& $20 \mathrm{~min}$ & $3.88^{*}$ & 1.21 & $-48 \%$ \\
\hline
\end{tabular}

* Significant change from baseline $(P<.05)$.

Table 3 Comparison of Maximum Wrist-Flexion Strength 20, 25, and $30 \mathrm{mil}$ After Baseline

\begin{tabular}{llccc}
\hline & & \multicolumn{2}{c}{ Wrist Flexion $(\mathbf{l b})$} & \\
\cline { 3 - 4 } Treatment & & \\
\cline { 3 - 4 } Menthol & Time from baseline & Mean maximum torque & SE & Change from 20 min \\
\cline { 3 - 4 } & $20 \mathrm{~min}$ & 10.86 & 0.96 & \\
\multirow{4}{*}{ Control } & $25 \mathrm{~min}$ & 11.11 & 1.14 & $2.27 \%$ \\
& $30 \mathrm{~min}$ & 10.82 & 1.13 & $-0.41 \%$ \\
& $20 \mathrm{~min}$ & 9.93 & 0.96 & \\
Ice & $25 \mathrm{~min}$ & 10.59 & 1.12 & $6.71 \%$ \\
& $30 \mathrm{~min}$ & 10.14 & 1.13 & $2.13 \%$ \\
& $20 \mathrm{~min}$ & 9.81 & 0.96 & \\
& $25 \mathrm{~min}$ & 10.23 & 1.14 & $4.26 \%$ \\
& $30 \mathrm{~min}$ & 10.25 & 1.13 & $4.47 \%$ \\
\hline
\end{tabular}

* Significant within-treatment change from 20-min measurement $(P<.05)$. 
Table 4 Comparison of Maximum Wrist-Extension Strength 20, 25, and 30 min After Baseline

\begin{tabular}{|llccc}
\hline & & \multicolumn{2}{c}{ Wrist Extension (lb) } & \\
\cline { 3 - 4 } Treatment & Time from baseline & Mean maximum torque & SE & Change from 20 min \\
\hline \multirow{2}{*}{ Menthol } & $20 \mathrm{~min}$ & 7.54 & 0.61 & \\
& $25 \mathrm{~min}$ & $8.17^{*}$ & 0.74 & $8.38 \%$ \\
& $30 \mathrm{~min}$ & $8.17^{*}$ & 0.74 & $8.38 \%$ \\
Control & $20 \mathrm{~min}$ & 7.11 & 0.61 & \\
& $25 \mathrm{~min}$ & $7.76^{*}$ & 0.74 & $9.14 \%$ \\
& $30 \mathrm{~min}$ & $7.88^{*}$ & 0.74 & $10.70 \%$ \\
Ice & $20 \mathrm{~min}$ & 7.17 & 0.61 & \\
& $25 \mathrm{~min}$ & 7.35 & 0.74 & $2.43 \%$ \\
& $30 \mathrm{~min}$ & 7.42 & 0.74 & $3.47 \%$
\end{tabular}

* Significant within-treatment change from 20 -min measurement $(P<.05)$.

over $8 \%$ under the menthol condition to nearly $11 \%$ in the control. Wrist-extension strength did not change over the 3 data-collection points under the ice condition.

\section{Discussion}

The results suggest support for the study hypotheses and are consistent with previous investigators who found that the application of ice or menthol gel results in decreased blood flow. The application of ice has long been used to reduce inflammation after a soft-tissue injury by decreasing blood flow to the affected area. ${ }^{12}$ Studies have shown that the application of ice results in a localized vasoconstrictor response, decreasing blood flow to the area ${ }^{13,14}$ and inhibiting the inflammatory response. ${ }^{15}$ The vasoconstrictor effects of ice can be attributed to 3 different mechanisms: release of norepinephrine from an increase in local sympathetic activity, which activates the arterial $\alpha_{2}$-adrenergic receptors ${ }^{16}$; an increase in rho kinase activity ${ }^{17}$; and decreases in endothelial nitric oxide production. All these mechanisms increase translocation of the $\alpha_{2}$-adrenergic receptors to the smooth muscle ${ }^{17-19}$ and increase calcium sensitization, ${ }^{17,20,21}$ which results in arterial constriction and reduced blood flow.

The results of this study indicate that blood flow through the radial artery appears to be significantly reduced within 5 minutes of applying a $3.5 \%$ menthol gel to the forearm. This reduction in blood flow with menthol was not sustained at 10,15 , or 20 minutes after treatment application. Ice applied to the forearm appears to reduce blood flow only at 20 minutes of application. Thus, the results of this study indicate that menthol appears to have a fast, short-acting effect of reducing blood flow to tissues distal to application, whereas ice reduces blood flow only at 20 minutes after sustained application. These differences in the time to affect blood flow may be attributable to the different mechanisms by which these 2 treatments do so. Previous authors have indicated that the reductions in blood flow resulting from ice therapy occur through decreases in local tissue temperature beginning 
at $24^{\circ} \mathrm{C}^{17}$ with a corresponding decrease in metabolic activity in the affected tissues. ${ }^{17-21}$ The results of this study indicate that somewhere between 15 and 20 minutes are required to achieve this effect of ice therapy in reducing blood flow to the underlying tissues.

In contrast, menthol gel may affect blood flow through neuronal mechanisms that respond faster to this treatment but are also quickly sensitized to this stimulation, resulting in an immediate but short-acting effect. ${ }^{22}$ Thus, because the reduced blood flow caused by menthol is mediated through neuronal mechanisms, the data support the effect of menthol as fast acting but brief in duration. The mechanism by which ice treatment reduces blood flow is through slowing down metabolic processes at the tissue level. The duration of time to evoke this response appears to be somewhere between 15 and 20 minutes, which is longer than the menthol treatment takes to achieve a similar reduction in blood flow. Somewhat surprising findings in this study were the significant increase in blood flow observed in the control condition and the high degree of variability in blood flow at baseline in each of the treatment conditions. There may be a variety of explanations for these observations. First, there is a great deal of variability in the literature in measurement of arterial blood flow at the wrist. Doppler measures of blood flow at the wrist including radial and ulnar arteries have ranged from $29.0 \pm 8.71$ to $25.7 \pm$ $9.97 \mathrm{~mL}$ expressed as pulse amplitudes. ${ }^{23}$ Second, peripheral blood flow can be affected by numerous factors that were beyond the control of the investigator, including hydration, emotional state, and characteristics of the ambient environment. A limitation of conducting this study with humans is that these factors cannot be easily controlled and may have contributed to the high degree of variability in the blood-flow measures. One possible explanation for the increase in blood flow under the control condition is that the data-collection protocol, which included palpating and touching the subjects' forearms, may have altered their heart rate or blood pressure or increased blood flow to their periphery. ${ }^{24,25}$ This increase in blood flow resulting from touching the subject further enhances the validity of the experimental interventions, which demonstrated significant declines in blood flow under the same conditions involving touching.

The results for wrist-flexion and -extension strength also partially support the second study hypothesis that the application of ice or menthol gel results in different measures of muscle strength. The effect of ice therapy on muscle performance has been studied, and there is general agreement that enzymatic processes are slowed with almost any level of cooling. Slowed enzymatic processes contribute to reduce muscle strength and power. A number of early studies in this area indicated that the application of ice had no effect on muscle strength but did increase endurance. ${ }^{26,27}$ In a recent study, muscle power and functional performance were found to be negatively affected immediately and 20 minutes after a 10-minute ice-bag application. ${ }^{28}$ In a review, Drinkwater ${ }^{29}$ concluded that the slowed chemical processes in the tissue and slowed nerve conduction resulting from ice therapy likely impair the rate of force development, reducing local muscle endurance during dynamic contractions and impairing manual dexterity. Thus, the effect of ice on muscle function and blood flow likely occurs locally as a result of decreased tissue temperature. ${ }^{30}$

Our findings that menthol may improve function appear attributable to previous investigators' observation that menthol stimulates TRP receptors classified as TRPM8, which are found mainly along thermoreceptive pathways. ${ }^{31-33}$ Menthol gels 
may also act as an analgesic to lessen pain by activating the TRP channel (TRPM8) located in sensory neurons. ${ }^{34}$ Furthermore, menthol appears to reduce pain through a selective activation of $\kappa$-opioid receptors. ${ }^{8}$ Our findings are consistent with one of the few double-blind studies examining the effects of menthol, in which McKay et $\mathrm{al}^{35}$ reported that a gel containing menthol was effective in relieving the pain and stiffness of osteoarthritis in patients without adverse effects. Kraemer et $\mathrm{al}^{36}$ also reported that a menthol gel improved the functional ability of patients with knee arthritis. These effects of menthol may be a result of mitigating pain during the activity rather than enhancing enzymatic activity of the muscle. Thus, it appears that the effects of menthol to improve function are neurological by inhibiting nociceptive sensory pathways that dampen muscle activation.

Consistent with these findings cited in the literature, in both the menthol and control conditions wrist-extension strength increased at the 25- and 30-minute assessment points compared with the 20 -minute assessment point. This increase may be attributable to familiarization with the protocol or the muscle warming up and becoming more efficient with the activity. ${ }^{37,38}$ This measure of strength did not increase over the 3 assessment points with the ice treatment. These findings support the conclusion of previous investigators ${ }^{28,29}$ that ice treatment reduces the metabolic activity of the underlying tissues and that this effect may continue for up to 10 minutes after removal of the ice.

The nonsignificant observation that the menthol treatment resulted in a trend toward higher wrist strength at each data-collection point than in the control and ice treatments may have clinical significance. Although it was not statistically significant because of insufficient statistical power, the menthol treatment consistently resulted in what appeared to be higher measures of wrist-flexion and -extension strength at all data-collection points This observation may be attributable to the previously reported counterirritant effect of menthol as a treatment for pain, which may have been elicited during the performance of the 30 maximum wrist-flexion and -extension exercises to assess muscle strength. ${ }^{39}$ Perhaps during these measures of maximum effort, the menthol treatment blocked pain impulses from the involved muscles resulting from lactic-acid buildup in the working muscles, which would normally inhibit the motoneurons innervating these muscles. A second possible explanation of the observed trend that the menthol treatment may result in higher levels of maximum wrist strength may be the counterirritant effect of menthol's blocking pain impulses from ascending to higher cortical levels. Fewer pain impulses from the working muscles may have resulted in the subjects' exerting more conscious effort during the assessments of wrist strength, which resulted in higher measures. These nonsignificant findings that menthol may have a performanceenhancing effect on maximal strength appear to merit further study.

The results of this study must be interpreted cautiously and are susceptible to numerous threats to internal and external validity. First, the interventions could not be blinded to the investigators or the participants because of the distinct odor of the menthol gel and the cold sensation of the ice. This lack of blinding may have resulted in a Hawthorne or placebo effect on the subjects' effort during the strength assessments. Second, blood flow was assessed in a single artery distal to the application of the experimental treatments, and the blood flow in the tissues directly adjacent to the treatments was not assessed. Thus, the effects of the interventions on the tissues most directly affected by the treatment were not measured. 
A third limitation of this study was that the mechanisms resulting in alterations in blood flow and muscle strength were not directly examined. The physiological basis for the observed effects of the interventions on the outcome variables can only be conjectured based on the findings of previous investigators. Finally, the small sample size likely limited the statistical power to detect a distinct treatment effect on blood flow and strength. Furthermore, the findings of this study may not be replicable among older populations with chronic health conditions that may affect blood flow and muscle strength.

\section{Conclusion}

In conclusion, it appears that topical application of a $3.5 \%$ menthol gel reduces blood flow in an artery distal to its application within 5 minutes after application but not 10 to 20 minutes after the application. Ice reduces blood flow in an artery distal to its application only after 20 minutes of application. Thus, ice is slower to reduce blood flow, and menthol results in more rapid but short-lasting reductions in blood flow. After the removal of ice, wrist-extension strength does not increase after repeated assessments of strength as it does during a no-treatment control condition and 20 minutes after application of 3.5\% menthol gel. Finally, further study is warranted as to the effects of topical menthol gel on performance of the underlying muscles.

\section{References}

1. Hubbard TJ, Denegar CR. Does cryotherapy improve outcomes with soft tissue injury? J Athl Train. 2004;39(3):278-279.

2. Esparza F, Cobian C, Jimenez JF, Garcia-Cota JJ, Sanchez C, Maestro A. Topical ketoprofen TDS patch versus diclofenac gel: efficacy and tolerability in benign sport related soft-tissue injuries. Br J Sports Med. 2007;41(3):134-139.

3. Kostopoulos D, Rizopoulos K. Effect of topical aerosol skin refrigerant (spray and stretch technique) on passive and active stretching. J Bodyw Mov Ther. 2008;12(2):96104.

4. Hubbard TJ, Aronson SL, Denegar CR. Does cryotherapy hasten return to participation? a systematic review. J Athl Train. 2004;39(1):88-94.

5. Ragan BG, Nelson AJ, Foreman JH, Bell GW, Iwamoto GA. Effects of a menthol-based analgesic balm on pressor responses evoked from muscle afferents in cats. Am J Vet Res. 2004;65(9):1204-1210.

6. Ichiyama RM, Ragan BG, Bell GW, Iwamoto GA. Effects of topical analgesics on the pressor response evoked by muscle afferents. Med Sci Sports Exerc. 2002;34(9):1440 1445.

7. Melzack R, Wall PD. Pain mechanisms: a new theory. Science. 1965;150(699):971-979.

8. Galeotti N, Di Cesare Mannelli L, Mazzanti G, Bartolini A, Ghelardini C. Menthol: a natural analgesic compound. Neurosci Lett. 2002;322(3):145-148.

9. Haeseler G, Maue D, Grosskreutz J, et al. Voltage-dependent block of neuronal and skeletal muscle sodium channels by thymol and menthol. Eur J Anaesthesiol. 2002;19(8):571-579.

10. Bleakley C, McDonough S, MacAuley D. The use of ice in the treatment of acute softtissue injury: a systematic review of randomized controlled trials. Am J Sports Med. 2004;32(1):251-261. 
11. Schlosser T, Veltmann C, Lohmaier S, et al. Determination of the renal blood flow in macro- and microcirculation by means of pulse inversion imaging. Rofo. 2004;176(5):724-730.

12. MacAuley D. Do textbooks agree on their advice on ice? Clin J Sport Med. 2001;11(2):67-72.

13. Kilgour RD, Carranza A, Findlay R. Suppression of the reactive hyperemic response in the forearm due to local hand cooling. Aviat Space Environ Med. 1997;68(1):46-50.

14. Hermann J. Cryotherapy. Z Rheumatol. 2009;68(7):539-541.

15. Swenson C, Sward L, Karlsson J. Cryotherapy in sports medicine. Scand J Med Sci Sports. 1996;6(4):193-200.

16. Hodges GJ, Zhao K, Kosiba WA, Johnson JM. The involvement of nitric oxide in the cutaneous vasoconstrictor response to local cooling in humans. J Physiol. 2006;574(Pt 3):849-857.

17. Bailey SR, Eid AH, Mitra S, Flavahan S, Flavahan NA. Rho kinase mediates coldinduced constriction of cutaneous arteries: role of alpha2C-adrenoceptor translocation. Circ Res. 2004;94(10):1367-1374.

18. Bailey SR, Mitra S, Flavahan S, Flavahan NA. Reactive oxygen species from smooth muscle mitochondria initiate cold-induced constriction of cutaneous arteries. Am J Physiol Heart Circ Physiol. 2005;289(1):H243-H250.

19. Chotani MA, Flavahan S, Mitra S, Daunt D, Flavahan NA. Silent alpha(2C)-adrenergic receptors enable cold-induced vasoconstriction in cutaneous arteries. Am J Physiol Heart Circ Physiol. 2000;278(4):H1075-H1083.

20. Somlyo AP, Somlyo AV. $\mathrm{Ca}^{2+}$ sensitivity of smooth muscle and nonmuscle myosin II: modulated by $\mathrm{G}$ proteins, kinases, and myosin phosphatase. Physiol Rev. 2003;83(4):1325-1358.

21. Batchelor TJ, Sadaba JR, Ishola A, Pacaud P, Munsch CM, Beech DJ. Rho-kinase inhibitors prevent agonist-induced vasospasm in human internal mammary artery. $\mathrm{Br}$ J Pharmacol. 2001;132(1):302-308.

22. Patel T, Ishiuji Y, Yosipovitch G. Menthol: a refreshing look at this ancient compound. J Am Acad Dermatol. 2007;57(5):873-878.

23. Lee HS, Chang BC, Heo YJ. Digital blood flow after radial artery harvest for coronary artery bypass grafting. Ann Thorac Surg. 2004;77(6):2071-2074.

24. Weerapong P, Hume PA, Kolt GS. The mechanisms of massage and effects on performance, muscle recovery and injury prevention. Sports Med. 2005;35(3):235-256.

25. Diego MA, Field T. Moderate pressure massage elicits a parasympathetic nervous system response. Int J Neurosci. 2009;119(5):630-638.

26. Verducci FM. Interval cryotherapy decreases fatigue during repeated weight lifting. $J$ Athl Train. 2000;35(4):422-426.

27. Kimura IF, Thompson GT, Gulick DT. The effect of cryotherapy on eccentric plantar flexion peak torque and endurance. J Athl Train. 1997;32(2):124-126.

28. Fischer J, Van Lunen BL, Branch JD, Pirone JL. Functional performance following an ice bag application to the hamstrings. J Strength Cond Res. 2009;23(1):44-50.

29. Drinkwater E. Effects of peripheral cooling on characteristics of local muscle. Med Sport Sci. 2008;53:74-88.

30. MacAuley D. Ice therapy: how good is the evidence? Int J Sports Med. 2001;22(5):379384.

31. Behrendt HJ, Germann T, Gillen C, Hatt H, Jostock R. Characterization of the mouse cold-menthol receptor TRPM8 and vanilloid receptor type-1 VR1 using a fluorometric imaging plate reader (FLIPR) assay. Br J Pharmacol. 2004;141(4):737-745.

32. McKemy DD, Neuhausser WM, Julius D, McKemy DD, Neuhausser WM, Julius D. Identification of a cold receptor reveals a general role for TRP channels in thermosensation. Nature. 2002;416(6876):52-58. 
33. Rohacs T, Lopes CM, Michailidis I, et al. PI(4,5)P2 regulates the activation and desensitization of TRPM8 channels through the TRP domain. Nat Neurosci. 2005;8(5):626-634.

34. Proudfoot CJ, Garry EM, Cottrell DF, et al. Analgesia mediated by the TRPM8 cold receptor in chronic neuropathic pain. Curr Biol. 2006;16(16):1591-1605.

35. McKay L, Gemmell H, Jacobson B, Hayes B. Effect of a topical herbal cream on the pain and stiffness of osteoarthritis: a randomized double-blind, placebo-controlled clinical trial. J Clin Rheumatol. 2003;9(3):164-169.

36. Kraemer WJ, Ratamess NA, Maresh CM, et al. A cetylated fatty acid topical cream with menthol reduces pain and improves functional performance in individuals with arthritis. J Strength Cond Res. 2005;19(2):475-480.

37. Girard O, Carbonnel Y, Candau R, Millet G. Running versus strength-based warm-up: acute effects on isometric knee extension function. Eur J Appl Physiol. 2009;106(4):573-581.

38. Worrell TW, Perrin DH. Hamstring muscle injury: the influence of strength, flexibility, warm-up, and fatigue. J Orthop Sports Phys Ther.1992;16(1), 12-8.

39. Jordt SE, McKemy DD, Julius D. Lessons from peppers and peppermint: the molecular logic of thermosensation. Curr Opin Neurobiol. 2003;13(4):487-492. 